\title{
Finite-temperature geometric properties of the Kitaev honeycomb model
}

\author{
Francesco Bascone, ${ }^{1,2,3}$ Luca Leonforte, ${ }^{1}$ Davide Valenti, ${ }^{1,4}$ Bernardo Spagnolo,,${ }^{1,5,6}$ and Angelo Carollo ${ }^{1,5}$ \\ ${ }^{1}$ Dipartimento di Fisica e Chimica, Group of Interdisciplinary Theoretical Physics and CNISM, Università di Palermo, Viale delle Scienze, \\ Edificio 18, I-90128 Palermo, Italy \\ ${ }^{2}$ Dipartimento di Fisica “E. Pancini”, Università di Napoli Federico II, Complesso Universitario di Monte S. Angelo, via Cintia, Edificio 6, \\ 80126 Napoli, Italy \\ ${ }^{3}$ Istituto Nazionale di Fisica Nucleare, Sezione di Napoli, Complesso Universitario di Monte S. Angelo, via Cintia, Edificio 6, \\ 80126 Napoli, Italy \\ ${ }^{4}$ IBIM-CNR Istituto di Biomedicina ed Immunologia Molecolare “Alberto Monroy”, Via Ugo La Malfa 153, I-90146 Palermo, Italy \\ ${ }^{5}$ Radiophysics Department, Lobachevsky State University of Nizhni Novgorod, 23 Gagarin Avenue, Nizhni Novgorod 603950, Russia \\ ${ }^{6}$ Istituto Nazionale di Fisica Nucleare, Sezione di Catania, Via S. Sofia 64, I-90123 Catania, Italy
}

(Received 13 February 2019; revised manuscript received 23 April 2019; published 30 May 2019)

\begin{abstract}
We study finite-temperature topological properties of the Kitaev's spin-honeycomb model in the vortex-free sector with the use of the recently introduced mean Uhlmann curvature. We employ an appropriate fermionization procedure to study the system as a two-band $p$-wave superconductor described by a Bogoliubov-de Gennes Hamiltonian. This allows us to study relevant quantities such as Berry and mean Uhlmann curvatures in a simple setting. More specifically, we consider the spin honeycomb in the presence of an external magnetic field breaking time-reversal symmetry. The introduction of such an external perturbation opens up a gap in the phase of the system characterized by non-Abelian statistics. The resulting model belongs to a symmetry-protected class, so that the Uhlmann number can be analyzed. We first consider the Berry curvature on a particular evolution line over the phase diagram. The mean Uhlmann curvature and the Uhlmann number are then analyzed by assuming a thermal state. The mean Uhlmann curvature describes a crossover effect as temperature rises. In the trivial phase, a nonmonotonic dependence of the Uhlmann number, as temperature increases, is reported and explained.
\end{abstract}

DOI: 10.1103/PhysRevB.99.205155

\section{INTRODUCTION}

Topological phase transitions (TPTs) have emerged as a major new paradigm, which eludes the ordinary Landau classification, where phases are characterized by local order parameters and symmetry breaking occurring across criticalities. Topological phases indeed are identified by integer-valued invariants that are constructed out of ground-states properties [1-4]. Topological systems have attracted a great deal of interest on account of their peculiar properties, ranging from topologically protected edge states [5], to quantized current [6-11], and excitations with exotic statistics [12-14]. There is already a vast literature concerning zero-temperature TPTs, where the systems are described by pure states, but few studies have been done in the direction of a consistent mixed-state generalization. An emerging field of research tries to define and classify topological properties of systems in a mixed-state scenario, in nonequilibrium dissipative models or in finitetemperature systems at equilibrium [15-24]. Some recent results have shown that it may be possible to characterize topological phases for thermal states [15-24]. Among these, particularly promising approaches are based on Uhlmann holonomies [17-19,22], which are a formal generalization of the Berry phase for mixed states $[25,26]$. The latter quantity is in fact one of the main ingredients of the topological phases in the pure-state case. In recent works [27-29], it was shown that a physical quantity related to the Uhlmann connection, called mean Uhlmann curvature (MUC), is able to provide interesting features about TPT in the mixed-state case, accounting for the effect of temperature at thermal equilibrium or for out-of-equilibrium conditions [30-38]. It was also shown that in two-dimensional (2D) symmetry-protected topological systems, it is possible to define a Uhlmann number, which is a direct generalization of the Chern number, used as a topological invariant describing different topological phases at zero temperature [29,39]. However, this sodefined Uhlmann number is only formally analog to the Chern number, since it is not a topological invariant and it can be noninteger.

Noteworthy works [17-19] claim that certain models may show sharp finite-temperature (and temperature-driven) topological phase transitions, by using suitable topological measures. This is a controversial issue [20,22], which partly motivates the definition of the Uhlmann number in [29], as the latter provides a measure which overcomes the inconsistencies suffered by the topological measures of $[17,19]$ and, at the same time, is bound to experimentally accessible quantities, such as susceptibilities and conductivity [29].

Recently, much effort has been devoted to the study of fault-tolerant quantum computation via topology [14,40-42]. In this context, the Kitaev honeycomb model [43], extensively studied only at zero temperature, shows a rich phase structure that allows both Abelian and non-Abelian anyonic excitations. Non-Abelian anyons are, in fact, a crucial building block of topological quantum computing, whereby quantum computation is performed by braiding of excitations. The main purpose 
of this work is to study the Kitaev's honeycomb model at finite temperature using the mean Uhlmann curvature as a main tool. The analysis of finite-temperature phase transitions is, in fact, especially important in the quantum computing framework since this would allow one to understand how the topological concepts can be used at finite temperature, allowing for better practical opportunities. The honeycomb model under consideration shows a phase diagram containing gapped and gapless phases. At first, we introduce an external magnetic field breaking time-reversal symmetry. In this way, the system belongs to the symmetry-protected class $D$, which is characterized by a + charge-conjugation-type symmetry and by the absence of time-reversal and chiral symmetries [4]. In this context, one can analyze the system through the Uhlmann number since the Chern number is the proper zerotemperature topological invariant of such a class. Furthermore, such an external perturbation allows for the existence of non-Abelian excitations and opens a gap in an otherwise gapless phase. One of the main results of this paper is the analysis of the Uhlmann number behavior in the trivial phase for small values of the temperature close to the critical point. We find a nonmonotonic behavior, noted earlier in [29], which seems to be a general feature of the class, which can be, in principle, observed experimentally. We also study the Berry curvature of the model, both numerically and analytically, in the absence of external magnetic field interactions as a limit when the external coupling tends to zero. This is necessary because in the vanishing external field case, the Berry curvature is zero and it is therefore necessary to extend the parameter space. We analyze the Berry curvature only in this case because the model becomes topologically intrinsic and the Uhlmann number is no longer the quantity of interest.

The paper is organized as follows. In Sec. II, we discuss the spin-honeycomb model and its phase diagram. We employ the fermionization procedure introduced in [44], which has the advantage to give a closed form of the ground state in a BCS form. With this technique, the system can be considered as a two-band $p$-wave topological superconductor and this allows for more convenient calculations and better understanding of the results. In Sec. III, we carry out the calculation of the Berry curvature for the ground state, which is unique in the planar geometry, both in the presence and in the absence of an external magnetic field. In Sec. IV, we calculate the mean Uhlmann curvature and the Uhlmann number to obtain a description of the system at finite temperature, generalizing the results for the Berry curvature and for the Chern number in the presence of an external magnetic field acting on the honeycomb lattice. Section $\mathrm{V}$ contains the concluding remarks.

\section{HONEYCOMB MODEL}

We will consider the Kitaev honeycomb model [43], which comprises spin- $1 / 2$ particles arranged on the vertices of a honeycomb lattice. This model can support a rich variety of topological behaviors, depending on the values of its couplings [45].

The Hamiltonian of the system can be written as follows:

$$
H=-\sum_{\alpha \in\{x, y, z\}} \sum_{i, j} J_{\alpha} K_{i j}^{\alpha},
$$

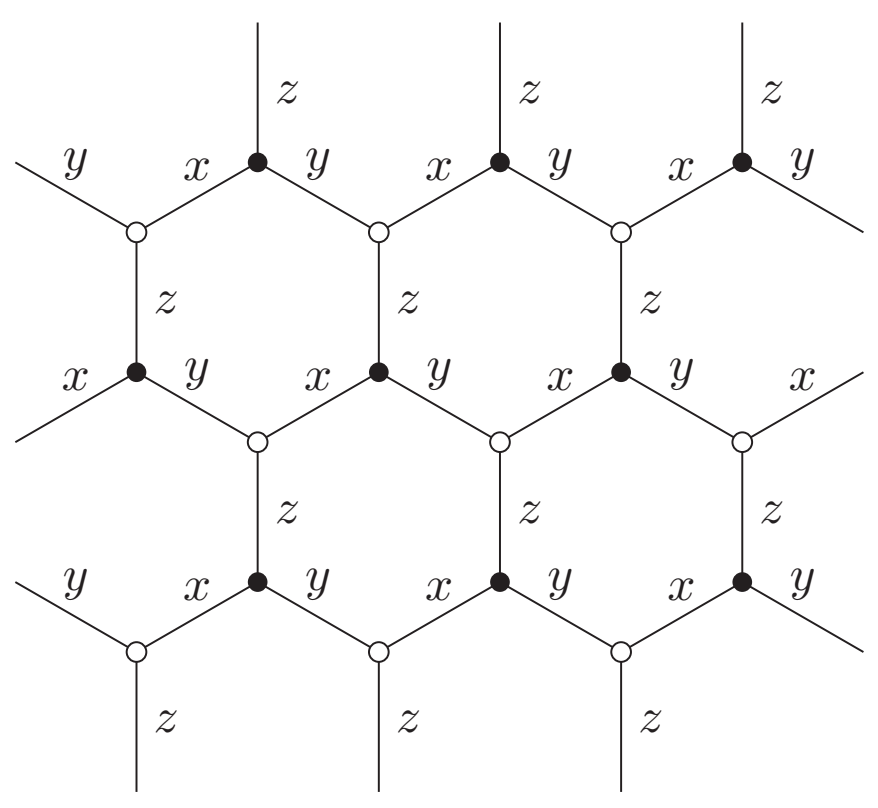

FIG. 1. Honeycomb lattice and link types.

with $K_{i j}^{\alpha}=\sigma_{i}^{\alpha} \sigma_{j}^{\alpha}$ denoting directional spin interaction between $i, j$ sites connected by $\alpha$ link (see Fig. 1), $J_{\alpha}$ are the dimensionless coupling coefficients of the two-body interaction, and the $\sigma_{i}^{\alpha}$ are the Pauli operators.

Products of $K$ operators can be used to construct loops on the lattice $K_{i_{1}, i_{2}}^{\alpha_{1}} K_{i_{2}, i_{3}}^{\alpha_{2}} \cdots K_{i_{n}, i_{1}}^{\alpha_{n}}$, and any loop constructed in this way commutes with all other loops and with the Hamiltonian. Therefore, these are good quantum numbers which provide a decomposition of the Hilbert space into the direct sum of invariant subspaces. In particular, the shortest loop symmetries are the plaquette operators,

$$
W_{p}=K_{12} K_{23} K_{34} K_{45} K_{56} K_{61}=\sigma_{1}^{x} \sigma_{2}^{y} \sigma_{3}^{z} \sigma_{4}^{x} \sigma_{5}^{y} \sigma_{6}^{z},
$$

where $p \in\{1,2, \ldots, m\}$ is a plaquette index and $m$ is the number of plaquettes. These $W_{p}$ operators represent loops around single hexagons and one way to visualize them is to look at the external link type that is connected to the vertices, e.g., in $\sigma_{1}^{x}$, for the external link-type $x$ connected with vertex 1 (Fig. 2).

The $W_{p}$ are a set of integrals of motion whose eigenvalues $\{ \pm 1\}$ identify different sectors of the Hilbert space. Each plaquette with $w_{p}=-1$ is said to carry a vortex, in analogy with the Ising $\mathbb{Z}_{2}$ gauge lattice theory. Therefore, each sector

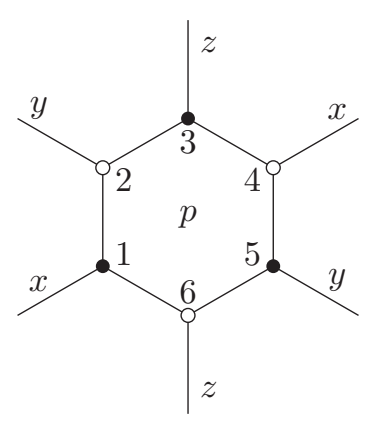

FIG. 2. Plaquette structure. 
corresponds to a particular choice of the string of eigenvalues over all the plaquettes, $\left.\left\{w_{p}\right\}\right|_{p \in\{1,2, \ldots, m\}}$.

In this way, the Hamiltonian can be decomposed as a direct sum over all the configurations,

$$
\mathcal{H}=\bigoplus_{\left\{w_{p}\right\}} \mathcal{H}_{\left\{w_{p}\right\}} .
$$

Thus, to solve the problem, one needs to find the eigenvalues of the Hamiltonian restricted to a particular sector. There are several ways to exactly solve this problem. According to the Kitaev's approach, the next step would be to map the spin degrees of freedom to the Majorana fermions and this will require each spin degree of freedom to be embedded in an extended Hilbert space of dimension four and then to obtain physical states as projections from the eigenstates of the extended Hamiltonian. However, in general, this is quite a daunting task. In some cases, however, it can be more convenient to explore an alternative route, first developed by $[44,46]$. The latter consists of a Jordan-Wigner (JW) fermionization procedure, mapping "hard-core" bosons operators to fermionic operators through string operators. This procedure allows for an explicit construction of the eigenstates of the system.

A theorem by Lieb [47] shows that the ground state of the system must lie in the vortex-free sector. By focusing on the vortex-free sector, in a planar lattice geometry, one can exploit the translational symmetry and use the Fourier transform to derive the energy spectrum. The aforementioned JW transformation results in the following Bogoliubov-de Gennes (BdG)-like Hamiltonian,

$$
H=\frac{1}{2} \sum_{\mathbf{q}}\left(C_{\mathbf{q}}^{\dagger}, C_{-\mathbf{q}}\right) H_{\mathbf{q}}\left(\begin{array}{c}
C_{\mathbf{q}} \\
C_{-\mathbf{q}}^{\dagger}
\end{array}\right),
$$

where

$$
H_{\mathbf{q}} \equiv\left(\begin{array}{cc}
\xi_{\mathbf{q}} & \Delta_{\mathbf{q}} \\
\Delta_{\mathbf{q}}^{*} & -\xi_{\mathbf{q}}
\end{array}\right)
$$

with

$$
\begin{aligned}
\xi_{\mathbf{q}} & =2 J_{x} \cos q_{x}+2 J_{y} \cos q_{y}+2 J_{z}, \\
\Delta_{\mathbf{q}} & =i \beta_{\mathbf{q}}=2 i J_{x} \sin q_{x}+2 i J_{y} \sin q_{y} .
\end{aligned}
$$

Here we deal with a Cartesian basis where $\mathbf{q} \equiv\left(q_{x}, q_{y}\right)$.

Thus, the Kitaev honeycomb model is mapped into a spinless fermionic BdG Hamiltonian. The Hamiltonians $H_{\mathbf{q}}$ can then be diagonalized via Bogoliubov rotation of the mode operators: $b_{\mathbf{q}}=u_{\mathbf{q}} C_{\mathbf{q}}-v_{\mathbf{q}} C_{-\mathbf{q}}^{\dagger}$, with

$$
\begin{gathered}
u_{\mathbf{q}}=\sqrt{\frac{1}{2}+\frac{\xi_{\mathbf{q}}}{2 \epsilon_{\mathbf{q}}}}=\sqrt{1+\frac{J_{z}}{\epsilon_{\mathbf{q}}}}, \\
v_{\mathbf{q}}=-i \sqrt{\frac{1}{2}-\frac{\xi_{\mathbf{q}}}{2 \epsilon_{\mathbf{q}}}}=-i \sqrt{1-\frac{J_{z}}{\epsilon_{\mathbf{q}}}},
\end{gathered}
$$

where we also defined $\epsilon_{\mathbf{q}}=\sqrt{\xi_{\mathbf{q}}^{2}+\left|\Delta_{\mathbf{q}}\right|^{2}}=\sqrt{\xi_{\mathbf{q}}^{2}+\beta_{\mathbf{q}}^{2}}$.

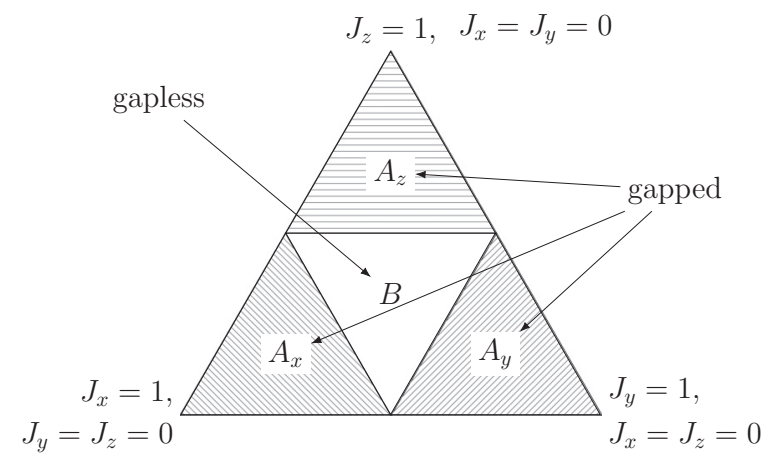

FIG. 3. Phase diagram of the honeycomb model: the triangle is the section of the positive octant by the plane $J_{x}+J_{y}+J_{z}=1$.

In terms of these operators, the diagonalized Hamiltonian takes the form

$$
H=\sum_{\mathbf{q}} \epsilon_{\mathbf{q}}\left(b_{\mathbf{q}}^{\dagger} b_{\mathbf{q}}-\frac{1}{2}\right),
$$

whose ground state has the BCS form

$$
\left|\Psi_{0}\right\rangle=\prod_{\mathbf{q}}\left(u_{\mathbf{q}}+v_{\mathbf{q}} C_{\mathbf{q}}^{\dagger} C_{-\mathbf{q}}^{\dagger}\right)|0\rangle,
$$

which is annihilated by all the $b_{\mathbf{q}}$. From the dispersion relation, it is possible to find the phase diagram structure of the system. One can readily check that the following triangular inequalities:

$$
\begin{aligned}
& \left|J_{x}\right| \leqslant\left|J_{y}\right|+\left|J_{z}\right|, \quad\left|J_{y}\right| \leqslant\left|J_{x}\right|+\left|J_{z}\right|, \\
& \left|J_{z}\right| \leqslant\left|J_{x}\right|+\left|J_{y}\right|,
\end{aligned}
$$

if satisfied, determine whether the spectrum is gapless. In Fig. 3, we explicitly depict the above triangular condition in the positive octant $\left(J_{x}, J_{y}, J_{z} \geqslant 0\right)$. One can easily derive the representation in the other octants, by symmetry. The triangular region in the phase diagram determined by the above conditions will be called the gapless $B$ phase, while the other three equivalent regions will be indicated as gapped $A$ phases.

\section{BERRY CURVATURE IN THE VORTEX-FREE SECTOR ON THE PLANE}

A standard object used to classify the properties of certain topological band-gap models in 2D is the Chern number, $C:=$ $\frac{1}{2 \pi} \iint_{B Z} d q_{x} d q_{y} \mathcal{F}_{q_{x}, q_{y}}$. As is well known, $C$ is derived from the Berry curvature with respect to the quasimomenta, i.e., $\mathcal{F}_{q_{x}, q_{y}}=\partial_{x} \mathcal{A}_{q_{x}}-\partial_{y} \mathcal{A}_{q_{y}}$, with $\mathcal{A}_{q_{i}}=-i\left\langle\Psi\left|\partial_{q_{i}}\right| \Psi\right\rangle$, where $\partial_{q_{i}}:=\partial / \partial_{q_{i}}$, with $i \in\{x, y\}$.

Notice, however, that in this section, we are not interested in the Chern number, but rather on the analytical properties of the curvature calculated with respect to the Hamiltonian parameters, across a phase transition. This is an alternative and well-established approach to study phase transitions from a purely geometrical point of view, rooted in the concepts of fidelity susceptibility and geometric information [48-50]. Within this approach, the object of interest is the Berry curvature, calculated with respect to the Hamiltonian parameters 
$\left\{J_{i}\right\}$, which is defined as

$$
\mathcal{F}_{i j}(J)=\partial_{i} \mathcal{A}_{j}-\partial_{j} \mathcal{A}_{i},
$$

where $\partial_{i}:=\partial / \partial J_{i}$, and the Berry connection is $\mathcal{A}_{i}(J)=$ $-i\left\langle\Psi\left|\partial_{i}\right| \Psi\right\rangle$, with $i \in\{x, y, z\}$.

We will calculate the curvature in Eq. (12) by focusing on the vertex-free configuration in a planar geometry. In this Hamiltonian sector, we will have to take into account only a single ground state, and therefore we will have to look at an Abelian Berry curvature.

Following the fermionization procedure used in [44], the Hamiltonian in Eq. (5) can be rewritten explicitly as

$$
H_{\mathbf{q}}=\mathbf{h}(J) \cdot \sigma,
$$

where $\mathbf{h}(J) \equiv\left(0,-\beta_{\mathbf{q}}, \xi_{\mathbf{q}}\right)$, and $\sigma$ are the Pauli matrices. The spectral Berry curvature (at fixed $\mathbf{q}$, the total curvature would be obtained summing over all momenta) is easily computed directly by means of the relation

$$
\mathcal{F}_{i j}=\frac{1}{2 h^{3}}\left[\left(\partial_{i} \mathbf{h}\right) \times\left(\partial_{j} \mathbf{h}\right)\right] \cdot \mathbf{h},
$$

where $h:=|\mathbf{h}|=\epsilon_{\mathbf{q}}$. One can readily check that this curvature appears to be zero everywhere, on account of the time-reversal (TR) and parity (P) symmetries of the model.

As discussed in Sec. I, adding a TR and/or P symmetrybreaking term in the Hamiltonian in the gapless $B$ phase, for instance by means of an external magnetic field, results in a nonvanishing gap opening up. This condition allows for the creation of non-Abelian anyonic excitation. Alternatively, one can add a three-body interaction term (TR and P symmetry breaking) of the form [51]

$$
H_{\mathrm{int}}=-\kappa \sum_{\mathbf{q}} \sum_{l=1}^{4} P_{\mathbf{q}}^{(l)},
$$

where $\kappa$ is the three-body external coupling, and with the second summation running over the four terms,

$$
\sum_{l=1}^{4} P_{\mathbf{q}}^{(l)}=\sigma_{1}^{x} \sigma_{6}^{y} \sigma_{5}^{z}+\sigma_{2}^{z} \sigma_{3}^{y} \sigma_{4}^{x}+\sigma_{1}^{y} \sigma_{2}^{x} \sigma_{3}^{z}+\sigma_{4}^{y} \sigma_{5}^{x} \sigma_{6}^{z} .
$$

The Hamiltonian $H_{\mathbf{q}}$ in Eq. (5) remains of the same form, provided a real part is added to $\Delta_{\mathbf{q}}: \Delta_{\mathbf{q}}=\alpha_{\mathbf{q}}+i \beta_{\mathbf{q}}$, with

$$
\alpha_{\mathbf{q}}=4 \kappa\left[\sin q_{x}-\sin q_{y}\right] .
$$

The diagonalized form of this Hamiltonian is then exactly the same as in Eq. (9), but with

$$
\epsilon_{\mathbf{q}}=\sqrt{\xi_{\mathbf{q}}^{2}+\left|\Delta_{\mathbf{q}}\right|^{2}}=\sqrt{\xi_{\mathbf{q}}^{2}+\alpha_{\mathbf{q}}^{2}+\beta_{\mathbf{q}}^{2}}
$$

We can still write $H_{\mathbf{q}}$ in the form of Eq. (13), but with a slightly different vector $\mathbf{h}(J) \equiv\left(\alpha_{\mathbf{q}},-\beta_{\mathbf{q}}, \xi_{\mathbf{q}}\right)$, and again calculate the spectral curvature. Of course, one should embed the threedimensional parameter manifold onto a four-dimensional one to include the extra parameter $\kappa$.

We find that the only nonvanishing components of the curvature in Eq. (14) are the $\mathcal{F}_{i \kappa}=-\mathcal{F}_{\kappa i}, i \in\{x, y, z\}$, which

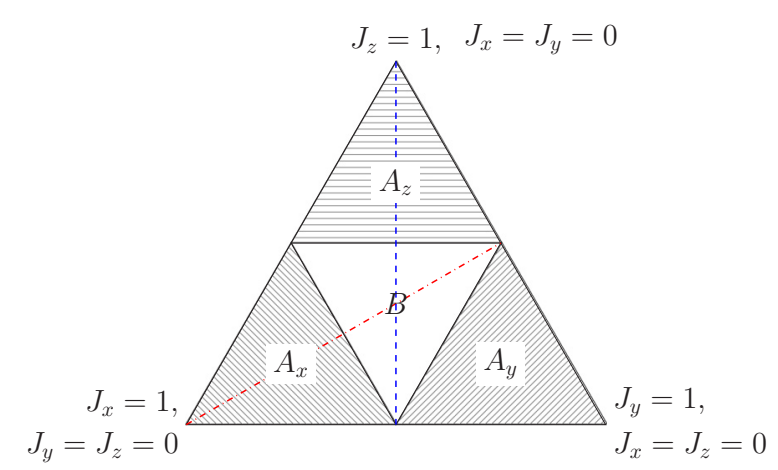

FIG. 4. Phase diagram: the blue dashed line, taken as the evolution line on which the Berry curvature is explored, is parametrized by $J_{x}=J_{y}=J$, while the red dot-dashed line is parametrized by $J_{y}=J_{z}$.

are explicitly given by

$$
\begin{aligned}
& \mathcal{F}_{x \kappa, \mathbf{q}}=\frac{\left[\sin q_{x}-\sin q_{y}\right]}{2 \epsilon_{\mathbf{q}}^{3}}\left[\xi_{\mathbf{q}} \sin q_{x}-\beta_{\mathbf{q}} \cos q_{x}\right], \\
& \mathcal{F}_{y \kappa, \mathbf{q}}=\frac{\left[\sin q_{x}-\sin q_{y}\right]}{2 \epsilon_{\mathbf{q}}^{3}}\left[\xi_{\mathbf{q}} \sin q_{y}-\beta_{\mathbf{q}} \cos q_{y}\right], \\
& \mathcal{F}_{z \kappa, \mathbf{q}}=-\frac{\left[\sin q_{x}-\sin q_{y}\right]}{2 \epsilon_{\mathbf{q}}^{3}} \beta_{\mathbf{q}} .
\end{aligned}
$$

In order to obtain the total curvature, the spectral curvature $\mathcal{F}_{i \kappa}$ needs to be summed over all quasimomenta $\mathbf{q}$ (or, in the thermodynamic limit, integrating over $d \mathbf{q}$ ).

Without loss of generality, let us choose the octant with $J_{i} \geqslant 0 \forall i \in\{x, y, z\}$. One sees that the three gapped phases $A_{i}$ are obtained for $J_{i}>J_{j}+J_{k}$, so that, for example, the region $A_{x}$ is determined by the condition $J_{x}>J_{y}+J_{z}$. The $B$ phase is instead realized by the conditions (11). The four phases are separated by quantum phase transition lines on which one of the $J_{i}$ is equal to the sum of the other two (see Fig. 3). A TR-P breaking perturbation [for instance, the term in Eq. (15) with $\kappa \neq 0$ ] opens up a gap in the otherwise gapless phase $B$. This would make both the $A$ 's and the $B$ phases gapped; however, a distinctive property of the latter, compared to former, is that the $A$ phases host Abelian excitations, whereas the lowenergy excitation of the $B$ phase satisfies non-Abelian anyonic statistics. Notice that in the chosen octant, the two phases are separated by the plane $J_{x}+J_{y}+J_{z}=1$, and independently of the phase we are in, the couplings have to satisfy such a normalization condition. To explore the behavior of the Berry curvature in the different phases and, in particular, on the transition lines between them, we can choose to study, without loss of generality, the system along the $J_{x}=J_{y}$ line, which basically vertically cuts the triangle diagram (blue dashed line in Fig. 4). With this choice of line cut, we can explore the dependence of the curvature in the $A_{z}$ and $B$ phases on $J_{z}$, with a special focus on the critical line at $J_{z}=\frac{1}{2}$. Due to the symmetry of the model, cutting along this line accounts for the qualitative behavior of the whole phase space. Under these conditions, we can use $J_{x}=J_{y}=J$ and, because of the normalization relation $J_{z}=1-2 J$, the curvature components are just expressed as functions of $0 \leqslant J \leqslant \frac{1}{2}$ along this line (the transition at $J_{z}=\frac{1}{2}$ is then realized at $J=\frac{1}{4}$ ). 
After these substitutions, the terms appearing in the expressions for the curvature components can be simplified as follows:

$$
\begin{aligned}
\alpha_{\mathbf{q}}= & 4 \kappa\left[\sin q_{x}-\sin q_{y}\right], \\
\beta_{\mathbf{q}}= & 2 J\left(\sin q_{x}+\sin q_{y}\right), \\
\xi_{\mathbf{q}}= & 2 J\left(\cos q_{x}+\cos q_{y}\right)+2-4 J, \\
\epsilon_{\mathbf{q}}= & \sqrt{\xi_{\mathbf{q}}^{2}+\alpha_{\mathbf{q}}^{2}+\beta_{\mathbf{q}}^{2}} \\
= & \left\{8 J^{2}\left[\cos \left(q_{x}-q_{y}\right)+1\right]+16 \kappa^{2}\left[\sin q_{x}-\sin q_{y}\right]^{2}\right. \\
& \left.+(2-4 J)\left[2+4 J\left(\cos q_{x}+\cos q_{y}-1\right)\right]\right\}^{1 / 2},
\end{aligned}
$$

so that the Berry curvature components in the thermodynamic limit get simplified as follows:

$$
\mathcal{F}_{i \kappa}(J)=\int_{-\pi}^{\pi} \int_{-\pi}^{\pi} d q_{x} d q_{y} \mathcal{F}_{i \kappa, \mathbf{q}}(J),
$$

with $i \in x, y, z$. Explicitly,

$$
\begin{aligned}
\mathcal{F}_{x \kappa, \mathbf{q}}= & 8\left(\sin q_{x}-\sin q_{y}\right) \\
& \times\left[J \sin \left(q_{x}-q_{y}\right)+(1-2 J) \sin q_{x}\right] \epsilon_{\mathbf{q}}^{-3}, \\
\mathcal{F}_{y \kappa, \mathbf{q}}= & 8\left(\sin q_{x}-\sin q_{y}\right) \\
& \times\left[J \sin \left(q_{y}-q_{x}\right)+(1-2 J) \sin q_{y}\right] \epsilon_{\mathbf{q}}^{-3}, \\
\mathcal{F}_{z \kappa, \mathbf{q}}= & 8 J\left(\sin ^{2} q_{y}-\sin ^{2} q_{x}\right) \epsilon_{\mathbf{q}}^{-3} .
\end{aligned}
$$

However, only one of the above expressions is independent. Indeed, $\mathcal{F}_{x \kappa}(J)=-\mathcal{F}_{y \kappa}(J)$, as can be seen by exchanging the dummy integration variables $q_{x} \rightarrow q_{y}$ under the integral, while we can see that $\mathcal{F}_{z \kappa}(J)=0$ by using the same argument. We can therefore limit our analysis to the $\mathcal{F}_{x \kappa}(J)$ component. This is an effect of the specific symmetry of the chosen cut line. Anyway, had we considered another line, we would have gotten similar results, but on a different set of components. For instance, if we cut the phase diagram from $A_{x}$ to the right angle of the $B$ phase (red dot-dashed line in Fig. 4), we get $\mathcal{F}_{y \kappa}(J)=-\mathcal{F}_{z \kappa}(J), \mathcal{F}_{x \kappa}(J)=0$, with $J_{y}=J_{z}=J$.

The numerical result of the integration along the line with $J_{x}=J_{y}=J$ for different values of $\kappa \neq 0$ is shown in Fig. 5. It is interesting to note that the function is peaked close to the criticality, at $J=\frac{1}{4}$, while it is regular enough over the whole region $0 \leqslant J \leqslant \frac{1}{2}$. However, for $\kappa \neq 0$, it is expected that the eventual criticality could not be evidenced by the Berry curvature, while they are surely caught by the Chern number. It is also worth noting that the vertical line in the phase diagram (see Fig. 4) is traveled downward, so that the $A_{z}$ phase is covered for $0 \leqslant J<\frac{1}{4}$, while the $B$ phase is covered for $\frac{1}{4}<J \leqslant \frac{1}{2}$.

The Berry curvature peak gets higher as $\kappa$ decreases to zero. This can be explained on account of the inverse dependence of the Berry curvature on the gap, which, in turn, tightens as $\kappa$ decreases. To analyze the $\kappa \rightarrow 0$ case, we study the Berry curvature numerically for small enough [52] values of $\kappa$, and we also study analytically the behavior of the curvature close to the transition line in the $\kappa \rightarrow 0$ limit, estimating the integrals around the Dirac points. This approach is justified by

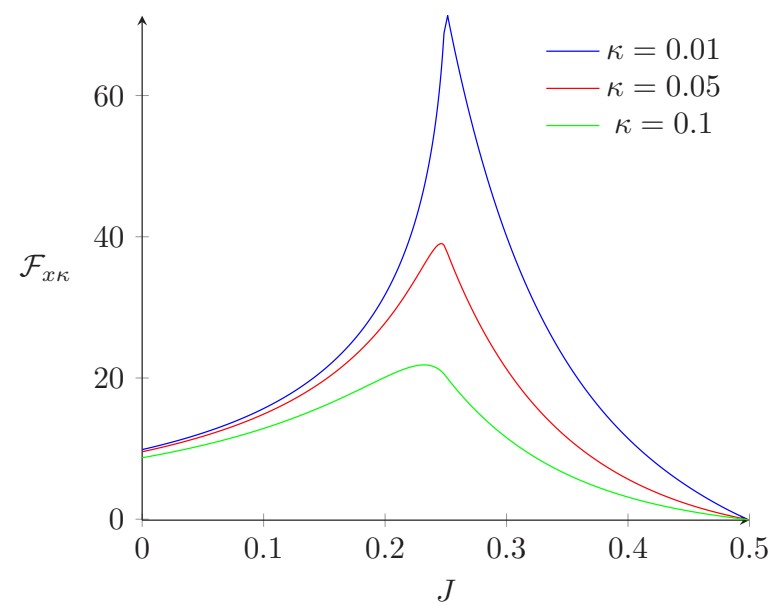

FIG. 5. $\mathcal{F}_{x \kappa}$ component of the Berry curvature as a function of $J$ along the evolution line $J_{x}=J_{y}=J, J_{z}=1-2 J$, with external coupling values $\kappa=0.01,0.05,0.1$.

the fact that the dominant contribution to the Berry curvature comes from the regions close to the Dirac points.

Therefore, the first thing to do is to find the minima of the energy spectrum around which the integrand function in Eq. (20) can be expanded (we consider again only the $\mathcal{F}_{x k}$ component). From the analysis of the the function $\epsilon_{\mathbf{q}}$, it follows that the two minima are found for the following values of the momentum components:

$$
q_{x}^{*}=-q_{y}^{*}= \pm \arccos \left[\frac{1-\frac{1}{2 J}}{1-\left(\frac{2 \kappa}{J}\right)^{2}}\right] .
$$

By performing a second-order expansion of the integrand function $\mathcal{F}_{x \kappa, \mathbf{q}}$ around these minima and using the eigenvalues of the Hessian matrix along the minimum eigendirections, we are left to compute the following integral:

$$
\int_{-R}^{R} \int_{-R}^{R} d x d y \frac{N_{0}+N_{1} x^{2}+N_{2} y^{2}}{\left(A^{2}+B^{2} x^{2}+C^{2} y^{2}\right)^{3 / 2}}=I_{0}+I_{1}+I_{2},
$$

with

$$
\begin{aligned}
N_{0} & =-\frac{8}{J^{2}}\left(J-\frac{1}{4}\right)(1-2 J)\left(\frac{2 \kappa}{J}\right)^{2}, \\
N_{1} & =\frac{-40}{J^{2}}\left(\frac{1}{2}-J\right)\left(J-\frac{1}{4}\right), \\
N_{2} & =\frac{8}{J^{2}}\left(\frac{1}{2}-J\right)\left(J-\frac{1}{4}\right), \\
A & =\frac{8 \kappa}{J} \sqrt{J-\frac{1}{4}}, \\
B & =4\left(\frac{1}{2}-J\right), \\
C & =4 \sqrt{J-\frac{1}{4}} .
\end{aligned}
$$

We also used the fact that the cross terms in the expansion are odd and they do not contribute in the symmetric integration region. The integration variables $x$ and $y$ are the 


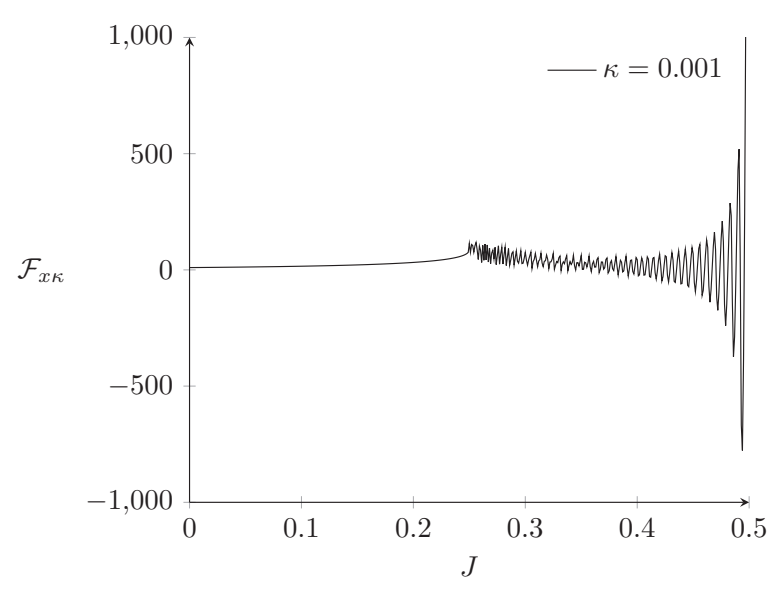

FIG. 6. $\mathcal{F}_{x \kappa}$ component of the Berry curvature as a function of $J$ along the evolution line $J_{x}=J_{y}=J, J_{z}=1-2 J$, with $\kappa=0.001$ to resemble the $\kappa \rightarrow 0$ case.

eigencoordinates, i.e., the momentum variables in the basis where the Hessian is diagonal. The finite integration radius $R$ is taken to enclose the minima and its explicit value is not important for the estimate. It is not hard to see that the contribution coming from $I_{0}=\int_{-R}^{R} \int_{-R}^{R} d x d y \frac{N_{0}}{\left(A^{2}+B^{2} x^{2}+C^{2} y^{2}\right)^{3 / 2}}$ vanishes in the $\frac{\kappa}{J} \rightarrow 0$ limit, while for the other two contributions, we find, in the same limit,

$$
\begin{aligned}
\mathcal{F}_{x} & =\lim _{\frac{\kappa}{J} \rightarrow 0}\left(I_{1}+I_{2}\right) \\
& \propto \frac{1}{J^{2}}\left[\frac{\ln \left(z+\sqrt{1+z^{2}}\right)}{z}-5 z^{2} \ln \left(\frac{1}{z}+\sqrt{1+\frac{1}{z^{2}}}\right)\right],
\end{aligned}
$$

with $z=\frac{\sqrt{J-\frac{1}{4}}}{\frac{1}{2}-J}$. The first thing to notice is that in the $J \rightarrow \frac{1}{4}$ limit, the Berry curvature is finite, which is in agreement with the numerical analysis.

However, even if there is no criticality, the Berry curvatures still give information about the different phases of the system. In fact, it can be seen numerically that for very small values of $\kappa$ resembling the $\kappa \rightarrow 0$ limit, we find very different behaviors below and above the transition line $J=\frac{1}{4}$. Namely, rapid oscillations appear in the nontrivial phase, as shown in Fig. 6, explicitly revealing the two different topological phases. Since the Berry curvature does not show any criticality, it is relevant to also analyze the first derivative of it (with respect to the parameter $J$ ). With a similar analysis, we can estimate the derivative of the curvature, obtaining the following result:

$$
\partial_{J} \mathcal{F}_{x \kappa} \propto \frac{\ln \left(J-\frac{1}{4}\right)}{J^{2}}
$$

which instead diverges to $-\infty$ in the $J \rightarrow \frac{1}{4}^{+}$limit, showing a criticality. The analytical behavior is in agreement with the numerical result, which, however, does not seem to be able to reveal the divergence in the transition point.

Therefore, the analysis of the Berry curvature at $\kappa \rightarrow 0$ shows a critical behavior, revealing the topological phase transition. This was not possible without expanding the parameter space.

\section{MEAN UHLMANN CURVATURE ESTIMATION AT FINITE TEMPERATURE}

In this section, we get a generalization of the Berry curvature to a finite-temperature case and, therefore, to a mixedstate representation.

Recently introduced in [27] was a proper mixed-state generalization of the Berry curvature, called mean Uhlmann curvature (MUC), which is gauge independent and which seems to properly describe topological phase transitions at finite temperature [27,29]. It was shown in [29] that for a two-level system described by a Hamiltonian of the form (13) in a finite-temperature equilibrium state described by the density operator $\rho=\frac{e^{-\beta H}}{Z}$, the MUC can be written as follows:

$$
\mathcal{U}_{i j}=\frac{\tanh ^{3}(\beta h)}{2 h^{3}}\left\{\left[\partial_{i} \mathbf{h}(J)\right] \times\left[\partial_{j} \mathbf{h}(J)\right]\right\} \cdot \mathbf{h}(J),
$$

where $\beta=\frac{1}{k T}$ and $Z$ is the partition function.

It is clear from Eq. (25) that in this case, the MUC is basically the Berry curvature as written in Eq. (14) with a further $[\tanh (\beta h)]^{3}$ factor, which ensures that in the $T \rightarrow 0$ limit, it reduces to the old pure-state Berry curvature, while in the high-temperature limit $(T \rightarrow+\infty)$, the MUC vanishes, as it should be. Indeed, the MUC can be seen as a kind of statistical average of the curvature of the states. Since the ground and excited states contribute with opposite curvature and at high temperatures the two states tend to be equally populated, it is expected to have $\mathcal{U} \rightarrow 0$.

In our case, we have a BdG-type Hamiltonian (5) and it was proven in [29] that in this particular case, the spectral MUC at fixed momentum $\mathbf{q}$ is given by a slightly different expression,

$$
\mathcal{U}_{i j, \mathbf{q}}=\frac{\tanh \frac{\beta h_{\mathbf{q}}}{2} \tanh ^{2} \beta h_{\mathbf{q}}}{2 h_{\mathbf{q}}^{3}}\left(\partial_{i} \mathbf{h}_{\mathbf{q}} \times \partial_{j} \mathbf{h}_{\mathbf{q}}\right) \cdot \mathbf{h}_{\mathbf{q}} .
$$

The difference is due to a different normalization condition. That is due to the fact that we are not really dealing with a two-level system, but this aspect was not effective in the Berry curvature expression because it was related to the ground state and only two states were involved.

\section{Uhlmann number}

It is also possible to define a so-called Uhlmann number in analogy with the Chern number,

$$
n_{U}:=\frac{1}{2 \pi} \iint_{B Z} d q_{x} d q_{y} \mathcal{U}_{q_{x}, q_{y}},
$$

with, in our case,

$$
\mathcal{U}_{q_{x}, q_{y}, \mathbf{q}}=\frac{\tanh \frac{\beta h_{\mathbf{q}}}{2} \tanh ^{2} \beta h_{\mathbf{q}}}{2 h_{\mathbf{q}}^{3}}\left(\partial_{x} \mathbf{h}_{\mathbf{q}} \times \partial_{y} \mathbf{h}_{\mathbf{q}}\right) \cdot \mathbf{h}_{\mathbf{q}},
$$

where the derivatives $\partial_{x}=\partial / \partial q_{x}, \partial_{y}=\partial / \partial q_{y}$ are with respect to the components of the quasimomentum. As discussed in [29], $n_{U}$ is only formally analog to the Chern number $C$ [53] since it is not purely topological and it can be noninteger. However, the two numbers are related by the zero-temperature limit, as it has to be: $\lim _{T \rightarrow 0} n_{U}=C$.

Similar measures of topology based on Uhlmann holonomy were introduced previously, with the aim of identifying 
finite-temperature phase transitions in 2D band-gap models [17-19]. However, as pointed out in [20], the above measures are generally ill defined, on account of the asymmetric role played by $q_{x}$ and $q_{y}$, and the inconsistencies obtained upon exchange of the latter. By contrast, the Uhlmann number cannot show any of these inconsistencies since the Uhlmann number is gauge invariant and symmetric in $q_{x}$ and $q_{y}$ by construction [27-29]. As a confirmation of this general statement, a look at Eq. (28) reveals that the integrand is symmetric under the exchange of $q_{x}$ and $q_{y}$.

Along the cut line specified in Sec. III, we have

$$
n_{U}=-\frac{J}{4 \pi} \iint_{B Z} d q_{x} d q_{y} \tanh \frac{\beta \epsilon_{\mathbf{q}}}{2} \tanh ^{2} \beta \epsilon_{\mathbf{q}} \frac{J \sin \left(q_{x}-q_{y}\right) \alpha_{\mathbf{q}}+2 \kappa \sin \left(q_{x}+q_{y}\right) \beta_{\mathbf{q}}+4 \kappa \cos q_{x} \cos q_{y} \xi_{\mathbf{q}}}{\epsilon_{\mathbf{q}}^{3}}
$$

where the $B Z$ is a torus (the momentum $\mathbf{q}$ is defined modulo the reciprocal lattice), and the $\alpha_{\mathbf{q}}(J), \beta_{\mathbf{q}}(J), \xi_{\mathbf{q}}(J), \epsilon_{\mathbf{q}}(J)$ functions are defined in Eq. (19).

The explicit form of $n_{U}$ is given by

$$
\begin{aligned}
n_{U}= & -\frac{J \kappa}{\pi} \iint_{B Z} d q_{x} d q_{y} \tanh \left(\frac{\beta \epsilon_{\mathbf{q}}}{2}\right) \tanh ^{2}\left(\beta \epsilon_{\mathbf{q}}\right) \\
& \times \frac{J\left[\cos q_{x}+\cos q_{y}\right]+\cos q_{x} \cos q_{y}(1-2 J)}{\left\{8 J^{2}\left[\cos \left(q_{x}-q_{y}\right)+1\right]+16 \kappa^{2}\left[\sin q_{x}-\sin q_{y}\right]^{2}+(2-4 J)\left[2+4 J\left(\cos q_{x}+\cos q_{y}-1\right)\right]\right\}^{1 / 2}} .
\end{aligned}
$$
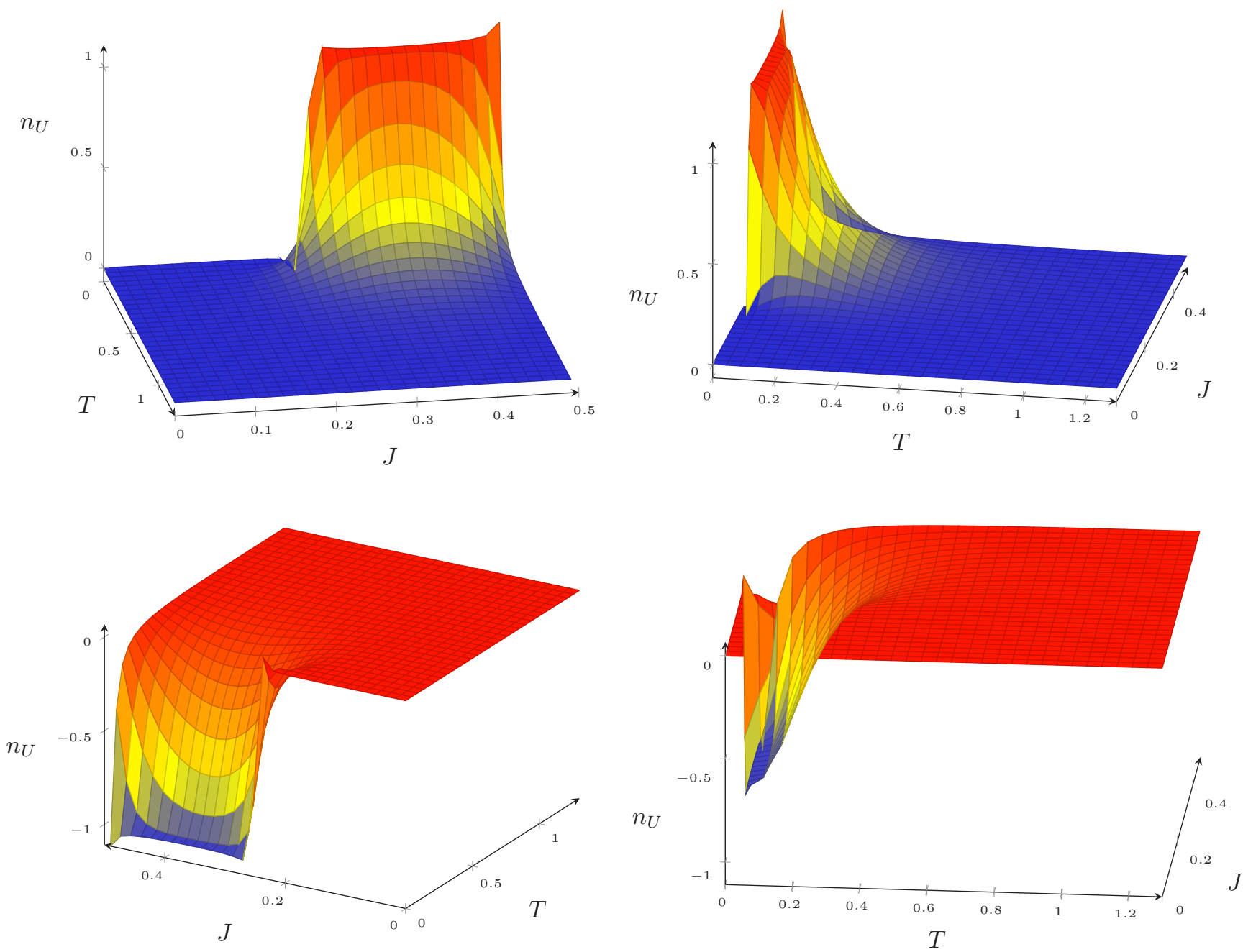

FIG. 7. The Uhlmann number $n_{U}$, as a function of $J$ and $T$ (in units of Boltzmann constant, $k_{B}=1$ ) along the cut line $J_{x}=J_{y}=J$, $J_{z}=1-2 J$, and two different values of the coupling parameter $\kappa$. First row: front (left) and side (right) view of the Uhlmann number with $\kappa=0.05$. Second row: front (left) and side (right) view with $\kappa=-0.05$ 


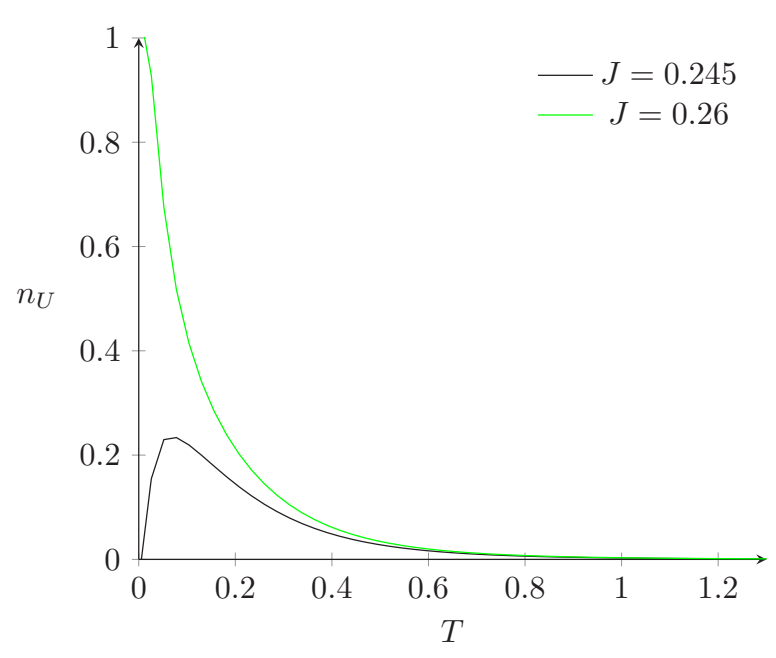

FIG. 8. Uhlmann number as a function of the temperature slightly below (green) and above (black) the transition point, with $\kappa=0.05$.

In Fig. 7 is shown the behavior of the Uhlmann number as a function of the evolution coupling parameter $J$ and temperature $T$, along the cut line $J_{x}=J_{y}=J, J_{z}=1-2 J$, for two values of the coupling constant $\kappa$, namely, $\kappa=0.05$ and $\kappa=-0.05$.

The first thing to notice is the $T \rightarrow 0$ behavior. The latter reproduces the Kitaev's result for the Chern number calculated by using the projection from the extended Hilbert space [43]. There, it was found that the Chern number is zero in the $A$ phase, which is topologically trivial, and \pm 1 in the $B$ phase. The sign of the Chern number appears to depend on a quantity, which in our case, is the sign of the external magnetic field coupling $\kappa$. Indeed, we find in our case that

$$
C=\left\{\begin{array}{l}
0, \quad A \text { phase } \\
1, \quad B \text { phase, } \kappa>0 \\
-1, \quad B \text { phase }, \kappa<0
\end{array}\right.
$$

It is important to note the nonmonotonic behavior of the Uhlmann number close to $J=\frac{1}{4}$ as a function of the temperature (see Fig. 8). Specifically, a peak appears for small values of the temperature in the $A$ phase. This effect was also present in the estimation of the Uhlmann number for a $p$-wave superconductor in [29] and seems to be a natural feature of the Uhlmann number. To explain this behavior of the Uhlmann number, we recall that we are working in a two-band system. As a consequence, in the topologically trivial phase $\left(0 \leqslant J<\frac{1}{4}\right)$, we have opposite contributions to the curvature, coming from the two bands close to the Dirac points and from the rest of them. Considering the valence band and expanding the Hamiltonian around a Dirac point, we find that the region close to this point contributes with $\mathrm{a} \pm c$ to the Uhlmann number, while the remaining region gives an opposite contribution $\mp c$. In turn, in the conduction band, we have the opposite situation. Indeed, at zero temperature, the only contribution comes from the valence band, and hence the sum of the two contributions coming from the Dirac points must give $n_{\left.U\right|_{T=0}}=0$. Increasing the temperature, we also get an additional contribution coming from the region of the conducting band close to the Dirac point. Therefore, the situation is not balanced and we have a positive net contribution to the Uhlmann number, which is represented by the peak shown in Fig. 8 (see black curve). At high temperature, the main contribution in the valence band comes from the region close to the Dirac point and the same happens for the conduction band. Since their curvature is opposite, the Uhlmann number goes back to zero. This can also be seen considering that since we are dealing with a two-level system, then $\mathcal{F}_{g}=-\mathcal{F}_{e}$, where $\mathcal{F}_{g}$ and $\mathcal{F}_{e}$ are the Berry curvature, respectively, for the valence band and for the conduction band. We know from Eq. (26) that (for the sake of simplicity, we will suppress every curvature component index and integration measure in the following)

$$
\mathcal{U}=w(\beta \epsilon) \mathcal{F}_{g}
$$

where

$$
\begin{aligned}
w(x) & =\tanh \left(\frac{x}{2}\right) \tanh ^{2}(x), \\
\mathcal{F}_{g} & =\frac{\left(\partial_{i} \mathbf{h}_{\mathbf{q}} \times \partial_{j} \mathbf{h}_{\mathbf{q}}\right) \cdot \mathbf{h}_{\mathbf{q}}}{2 \epsilon^{3}} .
\end{aligned}
$$

We can write the Uhlmann curvature as

$$
\mathcal{U}=f(\beta \epsilon) \mathcal{F}_{g}+g(\beta \epsilon) \mathcal{F}_{e},
$$

where

$$
\begin{aligned}
& f(x)=\tanh \left(\frac{x}{2}\right) \frac{1+\tanh ^{2}(x)}{2} \\
& g(x)=\tanh \left(\frac{x}{2}\right) \frac{1-\tanh ^{2}(x)}{2}
\end{aligned}
$$

so that

$$
n_{U}=\frac{1}{2 \pi} \int_{B Z} \mathcal{U}=\frac{1}{2 \pi}\left[\int f(\beta \epsilon) \mathcal{F}_{g}+\int g(\beta \epsilon) \mathcal{F}_{e}\right]
$$

Then, decomposing this in the contributions coming from the regions of the bands close and far from the Dirac point, a formal description is obtained. Indeed, decomposing the integration region as $B Z=\Omega_{c} \cup \Omega_{f}$, where $\Omega_{c}$ and $\Omega_{f}$ are the regions close and far from the Dirac point, we get

$$
\begin{aligned}
n_{U}= & \frac{1}{2 \pi}\left[\int_{\Omega_{c}} f(\beta \epsilon) \mathcal{F}_{g}+\int_{\Omega_{f}} f(\beta \epsilon) \mathcal{F}_{g}\right. \\
& \left.+\int_{\Omega_{c}} g(\beta \epsilon) \mathcal{F}_{e}+\int_{\Omega_{f}} g(\beta \epsilon) \mathcal{F}_{e}\right] .
\end{aligned}
$$

In the $\Omega_{f}$ region, for $T \gtrsim 0$, we can see that $g(\beta \epsilon) \approx 0$, while $f \approx 1$. Moreover, we can write $f(\beta \epsilon) \mathcal{F}_{g}=\tanh \left(\frac{\beta \epsilon}{2}\right) \mathcal{F}_{g}+$ $g(\beta \epsilon) \mathcal{F}_{e}$, and hence

$$
\begin{aligned}
n_{U} & =\frac{1}{2 \pi}\left[\int_{B Z} \tan \left(\frac{\beta \epsilon}{2}\right) \mathcal{F}_{g}+\int_{\Omega_{c}} g(\beta \epsilon) \mathcal{F}_{e}\right] \\
& =C+\frac{1}{2 \pi} \int_{\Omega_{c}} g(\beta \epsilon) \mathcal{F}_{e} .
\end{aligned}
$$

In the trivial phase case, $C=0$, but for low nonvanishing temperature, an additional positive term, which is responsible for the peak, is present. 
Finally, the green curve in Fig. 8 describes the Uhlmann number behavior just outside of the trivial phase, and it simply shows the standard expected behavior. This behavior is due to the additional term in Eq. (30), which is negative in this case [54].

\section{CONCLUSIONS}

After reviewing the Kitaev honeycomb model, we mapped the model Hamiltonian to a BdG one and gave explicit relations for the relevant quantities we were interested in. In particular, we assumed a translationally symmetric condition by considering the vortex-free sector of the model on an infinite plane. In Sec. III, we have calculated the Berry curvature by assuming an expanded parameter manifold, which included an extra time-reversal symmetry-breaking term (i.e., an effective magnetic field). This latter perturbation changes the classes of the model from an intrinsic topological material to a symmetry-protected topological material of class $D$. This was required both at an analytical and conceptual level: on the one hand, it allowed for an analytical headway for the calculation of the Berry curvature in the $\kappa \rightarrow 0$ limit, and on the other hand, it provided a way to properly assign a Chern number to the system and study the finite-temperature case by the Uhlmann number. For the $\kappa \rightarrow 0$ case, we estimated the Berry curvature by expanding around the relevant Dirac points. We found no criticality from it. However, the first derivative of the Berry curvature shows a divergence in the transition point that signals the phase transition. Therefore, the analysis of the Berry curvature in the $\kappa \rightarrow 0$ limit shows a criticality in the transition line that was not possible to estimate without a parameter expansion.

In Sec. IV, we calculated the mean Uhlmann curvature, as a generalization of the Berry curvature at finite temperature, and the Uhlmann number. Indeed, considering a thermal state, the analysis of the Uhlmann number makes it possible to understand how the topology of the honeycomb lattice model evolves as the temperature increases. In particular, no phase transition induced by the temperature is found, but it is shown that the nontrivial phase smoothly disappears at high temperatures. The zero-temperature limit correctly reproduces the Chern number result. We also found a nonmonotonic behavior of the Uhlmann number close to the criticality as a function of the temperature, with a peak appearing for small values of the temperature in the trivial phase. This peculiar nonmonotonic behavior can be observed only in regions which are close enough to the (zero-temperature) critical lines. The closer one gets to the critical line, the more pronounced this effect appears. This seems to be a general feature due to the partial filling of the conduction band.

\section{ACKNOWLEDGMENTS}

This work was supported by the Government of the Russian Federation through Agreement No. 074-02-2018-330 (2) and partially by the Ministry of Education and Research of Italian Government.
[1] A. Altland and M. R. Zirnbauer, Phys. Rev. B 55, 1142 (1997).

[2] A. P. Schnyder, S. Ryu, A. Furusaki, and A. W. W. Ludwig, Phys. Rev. B 78, 195125 (2008).

[3] S. Ryu, A. P. Schnyder, A. Furusaki, and A. W. W. Ludwig, New J. Phys. 12, 065010 (2010).

[4] C.-K. Chiu, J. C. Y. Teo, A. P. Schnyder, and S. Ryu, Rev. Mod. Phys. 88, 035005 (2016).

[5] Y. Hatsugai, Phys. Rev. Lett. 71, 3697 (1993).

[6] K. V. Klitzing, G. Dorda, and M. Pepper, Phys. Rev. Lett. 45, 494 (1980).

[7] D. J. Thouless, M. Kohmoto, M. P. Nightingale, and M. den Nijs, Phys. Rev. Lett. 49, 405 (1982).

[8] D. J. Thouless, Phys. Rev. B 27, 6083 (1983).

[9] Q. Niu and D. J. Thouless, J. Phys. A Gen. Phys. 17, 2453 (1984).

[10] S. Nakajima, T. Tomita, S. Taie, T. Ichinose, H. Ozawa, L. Wang, M. Troyer, and Y. Takahashi, Nat. Phys. 12, 296 (2016).

[11] D. C. Tsui, H. L. Stormer, and A. C. Gossard, Phys. Rev. Lett. 48, 1559 (1982).

[12] R. B. Laughlin, Phys. Rev. Lett. 50, 1395 (1983).

[13] D. P. Arovas, J. R. Schrieffer, and F. Wilczek (unpublished).

[14] C. Nayak, S. H. Simon, A. Stern, M. Freedman, and S. Das Sarma, Rev. Mod. Phys. 80, 1083 (2008).

[15] J. E. Avron, M. Fraas, G. M. Graf, and O. Kenneth, New J. Phys. 13, 053042 (2011).

[16] C.-E. Bardyn, M. A. Baranov, C. V. Kraus, E. Rico, A. İmamoǧlu, P. Zoller, and S. Diehl, New J. Phys. 15, 085001 (2013).
[17] Z. Huang and D. P. Arovas, Phys. Rev. Lett. 113, 076407 (2014).

[18] O. Viyuela, A. Rivas, and M. A. Martin-Delgado, Phys. Rev. Lett. 112, 130401 (2014).

[19] O. Viyuela, A. Rivas, and M. A. Martin-Delgado, Phys. Rev. Lett. 113, 076408 (2014).

[20] J. C. Budich and S. Diehl, Phys. Rev. B 91, 165140 (2015).

[21] D. Linzner, L. Wawer, F. Grusdt, and M. Fleischhauer, Phys. Rev. B 94, 201105(R) (2016).

[22] B. Mera, C. Vlachou, N. Paunković, and V. R. Vieira, Phys. Rev. Lett. 119, 015702 (2017).

[23] F. Grusdt, Phys. Rev. B 95, 075106 (2017).

[24] C.-E. Bardyn, L. Wawer, A. Altland, M. Fleischhauer, and S. Diehl, Phys. Rev. X 8, 011035 (2018).

[25] A. Uhlmann, Rep. Math. Phys. 24, 229 (1986).

[26] A. Uhlmann, Lett. Math. Phys. 21, 229 (1991).

[27] A. Carollo, B. Spagnolo, and D. Valenti, Sci. Rep. 8, 9852 (2018).

[28] A. Carollo, B. Spagnolo, and D. Valenti, Entropy 20, 485 (2018).

[29] D. P. Arovas, J. R. Schrieffer, F. Wilczek, Phys. Rev. Lett. 53, 722 (1984).

[30] L. Magazzù, D. Valenti, A. Carollo, and B. Spagnolo, Entropy 17, 2341 (2015).

[31] L. Magazzù, A. Carollo, B. Spagnolo, and D. Valenti, J. Stat. Mech. (2016) 054016.

[32] B. Spagnolo, A. Carollo, and D. Valenti, Entropy 20, 226 (2018). 
[33] D. Valenti, A. Carollo, and B. Spagnolo, Phys. Rev. A 97, 042109 (2018).

[34] B. Spagnolo, A. Carollo, and D. Valenti, Eur. Phys. J. Spec. Top. 227, 379 (2018).

[35] C. Guarcello, D. Valenti, A. Carollo, and B. Spagnolo, J. Stat. Mech. (2016) 054012.

[36] C. Guarcello, D. Valenti, A. Carollo, and B. Spagnolo, Entropy 17, 2862 (2015).

[37] B. Spagnolo, C. Guarcello, L. Magazzù, A. Carollo, D. Persano Adorno, and D. Valenti, Entropy 19, 20 (2017).

[38] B. Spagnolo, D. Valenti, C. Guarcello, A. Carollo, D. Persano Adorno, S. Spezia, N. Pizzolato, and B. Di Paola, Chaos, Solitons and Fractals 81, 412 (2015).

[39] Y. He, H. Guo, and C.-C. Chien, Phys. Rev. B 97, 235141 (2018).

[40] P. Shor, in Proceedings of the 37th Annual Symposium Foundations of Computer Science (IEEE Comput. Soc. Press, Los Alamitos, CA, 1996), pp. 56-65.

[41] A. Y. Kitaev, Ann. Phys. (NY) 303, 2 (2003).

[42] A. Kitaev, in Advance in Theoretical Physics: Landau Memorial Conference, edited by V. Lebedev and M. Feigel'man, AIP Conf. Proc. No. 1134 (AIP, New York, 2009) pp. 22-30.
[43] A. Kitaev, Ann. Phys. (NY) 321, 2 (2006).

[44] G. Kells, J. K. Slingerland, and J. Vala, Phys. Rev. B 80, 125415 (2009).

[45] V. Lahtinen, G. Kells, A. Carollo, T. Stitt, J. Vala, and J. K. Pachos, Ann. Phys. (NY) 323, 2286 (2008).

[46] G. Kells, A. T. Bolukbasi, V. Lahtinen, J. K. Slingerland, J. K. Pachos, and J. Vala, Phys. Rev. Lett. 101, 240404 (2008).

[47] E. H. Lieb, Phys. Rev. Lett. 73, 2158 (1994).

[48] A. C. M. Carollo and J. K. Pachos, Phys. Rev. Lett. 95, 157203 (2005).

[49] L. Campos Venuti and P. Zanardi, Phys. Rev. Lett. 99, 095701 (2007).

[50] S.-J. Gu, Int. J. Mod. Phys. B 24, 4371 (2010).

[51] G. Kells, V. Lahtinen, and J. Vala, Phys. Rev. B 89, 075122 (2014).

[52] The values are just big enough to avoid numerical instabilities.

[53] In our case, this Chern number can be expressed as $C=$ $\frac{1}{2 \pi} \iint_{B Z} d q_{x} d q_{y} \frac{1}{2 h_{\mathbf{q}}^{3}}\left[\frac{\partial}{\partial q_{x}} \mathbf{h}_{\mathbf{q}}(J) \times \frac{\partial}{\partial q_{y}} \mathbf{h}_{\mathbf{q}}(J)\right] \cdot \mathbf{h}_{\mathbf{q}}(J)$.

[54] In general, the sign of this term coming from the partial filling of the conduction band is always the opposite of that of the Chern number. 